Témoigner Témoigner. Entre histoire et mémoire

Getuigen Revue pluridisciplinaire de la Fondation Auschwitz

$126 \mid 2018$

Questions sur l'avenir du travail de mémoire

\title{
Les justes parmi les Nations dans un contexte pédagogique : quelques réflexions à partir du cas suisse
}

De Rechtvaardigen onder de naties in een pedagogische context Het geval

Zwitserland

\section{François Wisard}

\section{(2) OpenEdition \\ Journals}

\section{Édition électronique}

URL : https://journals.openedition.org/temoigner/7160

DOI : $10.4000 /$ temoigner.7160

ISSN : 2506-6390

Traduction(s) :

De Rechtvaardigen onder de naties in een pedagogische context - URL : https:// journals.openedition.org/temoigner/7192 [nl]

Éditeur :

Éditions du Centre d'études et de documentation Mémoire d'Auschwitz, Éditions Kimé

Édition imprimée

Date de publication : 2 avril 2018

Pagination : 60-67

ISBN : 978-2-930953-06-9

ISSN : 2031-4183

Référence électronique

François Wisard, «Les justes parmi les Nations dans un contexte pédagogique : quelques réflexions à partir du cas suisse », Témoigner. Entre histoire et mémoire [En ligne], 126 | 2018, mis en ligne le 20 janvier 2022, consulté le 04 février 2022. URL : http://journals.openedition.org/temoigner/7160 ; DOI : https://doi.org/10.4000/temoigner.7160 


\section{LesJustes parmi les Nations dans un contexte pédagogique : quelques réflexions à partir du cas suisse}

$\rightarrow$ François Wisard

L'historien suisse François Wisard dirige, depuis Service historique du Département fédéral des Affaires étrangères Berne. Il représente la Suisse dans le groupe de travail mémoriaux et musees de International
Holocaust Remembrance Alliance (IHRA). Il est lauteur de louvrage lauteur de louvrage
suivant: Les Justes suisses.

(1) Ministry of Foreign Affairs
of Portugal, Spared lives. The Actions of Three Portuguese Diplomats in World War Il,
Documentary exhibition, 2000 (2) Ainsi, jusqu'en 1995 : Anne-
Marie Im Hof-Piguet, La Filière en France occupée 1942-1944, Jacqueline Veuve, La filière, (film documentaire), 1987. Alexander Carl Lutz und seine Budapester Aktion, Geschichte und Porträt
Wald, Im Waldqut, 1986. Arieh Wald, Im Waldgut, 1986 . Arieh
Ben-Tov, Facing the Holocaust in Budapest: The International
Committee of the Red Cross and the Jews in Hungary, 1943-1945, Nijhoff/Norwell, 1988. Sebast Steiger. Die Kinder von Schloss
La Hille Basel Brunnen-Verlag

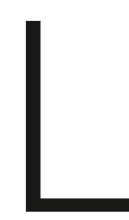

pose quelques réflexions générales et question sur les Justes parmi les Nations à partir d'un exemple concret, celui de la Suisse.

Rappelons que les Justes parmi les Nations sont des personnes non juives distinction, qui peutégalement être décernée à titre posthume, honore des hommes et des femmes qui ont sauvé des Juifs durant la Shoah d'une part sans recherche d'intérêt financier, d'autre part en mettant en danger leur vie ou leur liberté.

La place qu’occupe aujourd'hui le thème des Justes, et plus généralement celui du sauvetage des Juifs, dans le travail de mémoire sur la Shoah est assurément
importante (voir notamment Gensburger, 2015, p. 537-555). Au ler janvier 2017, le importante (voir notamment Gensburger, 2015, p. 537-555). Au $1^{\mathrm{er}}$ janvier 2017, le titre a été décerné à 26513 personnes à travers le monde. Ce nombre a fortement augmenté ces dernières décennies. Environ deux tiers des titres décernés depuis un peu plus de 50 ans l'ont été ces 25 dernières années. On relèvera aussi que les expositions sur l'histoire de la Shoah comportent désormais presque toujours une section sur les efforts de sauvetage de Juifs. Il faut signaler les ouvrages ou projets initiés par des ministères des Affaires étrangères pour mettre en valeur les actions d'anciens diplomates, comme au Portugal et en Espagne en $2000^{1}$ (Baer et Correa d'ancies derrea Martin-Argen en Suède et en Hongrie autour de Raoul Wallenberg en 2012, annee du centenaire de sa naissance. De telles utilisations comportent toutefois le risque d'instrumentaliser à des fins politiques les actions de sauvetage menées par des diplomates.

En Suisse, quelle place les Justes ont-ils occupée et occupent-ils encore aujourd'hui dans le travail de mémoire? Comme nous le verrons, la notion de « Juste suisse » soulève une série de questions, au même titre du reste que celle de « Juste français» ou de « Juste polonais», pour ne prendre que deux exemples parmi d'autres.

Pour l'heure, précisons simplement que Yad Vashem a honoré une septantaine de personnes qui possédaient la nationalité suisse durant la Shoah. Dès les

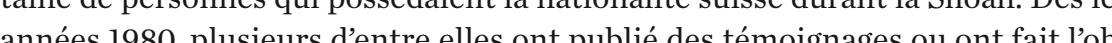
jet de livres ou de films documentaires ${ }^{2}$. Néanmoins, aucune entrée « Juste » ne figure parmi les quelque 40000 articles du Dictionnaire historique de la Suisse, une entreprise collective mise sur pied à cette époque-là ${ }^{3}$. À la fin du siècle dernier, le destin de ces Justes, qui commençait à être connu, n'était pas encore entré dans la mémoire collective.

Deux phénomènes témoignent d'un changement de situation:l'intérêt toujours intact pour les deux figures emblématiques des « Justes suisses » et l'apparition de matériel pédagogique spécifique.

Carl Lutz (1895-1975), un diplomate suisse en posteà Budapest, et Paul Grüninger (1891-1972) commandant de la police du canton frontalier de Saint-Gall au moment de l'Anschluss de l'Autriche, sont assurément les deux figures de « Justes suisses " qui dépassent le cadre national. Ils ne sont pas pour autant « exemplaires » de ces Justes, ainsi que nous le verrons lors de la présentation de leurs actions.

Même si leur destin était déjà bien connu et documenté, tant Lutz que Grüninger viennent de faire l'objet de nouveaux films et de nouveaux livres. Diffusé en 2013, le film Akte Grüninger ${ }^{4}$ a été réalisé 16 ans après un premier documentaire, lui-même basé sur un ouvrage qui avait été à lorigine de la réhabilitation de Grüninger dans la première moitié des années $1990^{5}$.

Pour Lutz, l'intérêt récent - nullement synonyme de renouvellement scientifique - est encore plus marqué. À un premier livre en 1988 ont succédé l'ouvrage de référence de Theo Tschuy en 1995, traduit en plusieurs langues, puis un premier film dix ans plus tard ${ }^{6}$. Ceci n’a pas empêché récemment un développement notable : deux nouveaux livres, un troisième en préparation, une exposition itinérante de la Fondation Lutz à Budapest inaugurée en 2008 au siège de l'ONU à New York et présentée depuis lors dans une dizaine, sinon même une vingtaine de villes? ${ }^{7}$. A cette liste, il convient d'ajouter un deuxième film, réalisé en 2014. De manière symptomatique, il porte le titre Carl Lutz, der vergessene Held et son réalisateur suisse a avoué qu'il venait de découvrir ce " héros oublié » (Mediendossier, 2014, p. 10).

Le domaine des matériels pédagogiques témoigne lui aussi d'un regain d'intérêt pour les «Justes suisses ». En 2003 les éditions de la Haute école pédagogique de Zurich publiaient un ouvrage sur les sauveteurs et Justes allemands, réalisé principalement - il est intéressant de le relever - à partir d'interviews conduits dans les années $1960^{8}$. Trois ans plus tard, une organisation juive basée en Suisse romande recherchait un auteur pour réaliser un deuxième ouvrage à vocation pédagogique, consacré aux Justes suisses, après une brochure sur l'ancien centre d'extermination d'Auschwitz-Birkenau. L'ouvrage fut publié en $2007^{9}$. Enfin, le canton de Lucerne en Suisse centrale, qui reste particulièrement actif dans l'organisation de la Journée internationale dédiée à la mémoire des victimes de la Shoah a consacré sa dernière édition (2015) d̀ des femmes et des hommes ayant fait preuve decoura civilduère la Shoah, mais aussi après 1945. Cinq Justes de nationalité suisse sont ainsi presentés

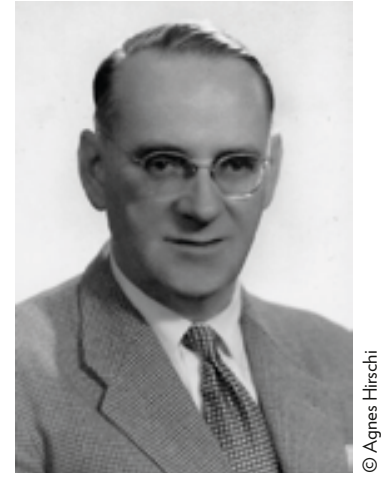
de la légation de Suisse en Hongrie en 1944-1945, Juste parmi les Nations en 1964

1992. Stefan Keller, Grüningers
Fall: Geschichten von Flucht und Hilfe, Zürich, Rovin 1994. Friedel Bohny-Reiter, Genève, Zoé, 1993. Theo Tschuy Carl Lutz und die Juden von
Budapest Zürich Verlag Ne Budapest, Zürich, Verlag Neue
Zürcher Zeitung, 1995. Marcel
Pasche, Années de guerre et de fasche, Anneess de guerre et de
fraternite, Le Mont-sur-Lausanne

(3) www.hls-dhs-dss.ch.

(4) Film d'Alain Gsponer (www. quuun des protagonistes est un

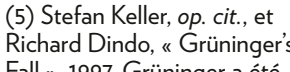
Fall, , 1997. Grüninger a etéé
démis de ses fonction en 1939 . puis condamné pénalement en 1940. La réhabilititation au plan (6) Alexander Grossman, op.

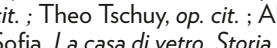
di Carl Lutz, lo svizzero che sallò̀ 62000 e errei, Locarno
Rezzonico, 2004 .

(7) György Vámos, Carl Lutzz
(1895-1975). Diplommate suisse à Budapesten 1944 . Un Iuste parmi les Nations, Gollion, Infolio, 2012.
Erika Rosenberg, Carl Lutz und 
Dans le travail de mémoire sur la Shoah, le thème des Justes, et plus généralement celui du sauvetage des Juifs, occupe aujourd'hui une place importante, tant en Suisse quà l'étranger. Cette contribution ne vise toutefois pas à questionner l'importance de cette place, à plaider pour son extension ou sa réduction. Elle examine les chances, et surtout les risques liés à l'utilisation de la figure de Justes dans un contexte pédagogique. La contribution propose une réflexion critique sur le contenu d'un certain travail de mémoire et non pas une recommandation sur l'ampleur de ce même travail Cette reflexion critique sera articulće autour de trois question

Convient-il de se limiter aux Justes oudexan vetage?

La notion de « Juste suisse », ou celle de « Juste français », est-elle évidente et pertinente?

- Existe-t-il des figures exemplaires de Justes parmi les nations?

CONVIENT-IL DE SE LIMITER AUX JUSTES OU

D'EXAMINER PLUS GLOBALEMENT LE SAUVETAGE?

Les Justes constituent-ils une catégorie utile dans un contexte pédagogique ? die Rettung ungarischer Juden vor
dem Holocaust, Munich, Herbig, dem Holocaust, Munich, Herbig,
2016 . Agnes Hirschi et Charlotte
Schallié, (éd.). Under Swiss Protection: Jewish Eyewitness Accounts from Wartime 2017. Foundation Lutz, Carl Lut and the Legendary Glass House in Budapest (exposition) (www
uveghaz.org). Contrairement
a Grüninger, Lutz ná jamais à Grüninger, Lutz nả jamais
été démis de ses fonctions
ni condamné. Voir notamment Marco Jorio, , Judenretter Carl
Lutz: Wurde er in der Schweiz Lutz: Wurde er in der Schwwerthlich getadelt, gestraft tatsächlich getadelt, gestraft
und vergessen, wie immer wieder behauptet wirl?", NZZ
Geschichte Der Revolutionär, Geschichte Der Revolutionär,
$2016, n^{\circ} 7$, octobre 2016, p. 120 (8) Beate Kosmala et LudewigDeutsche Retterinnen und Retter während des Holocaust,
Pestalozzianum, 2003. (9) François Wisard, Les Justes masses. Des actees de courage
meconnus au temps dela Shooah CICAD, 2007

(10) Daniel Von Aarburg, op. cit (11) Les autres exemples cités
ci-apress sont tirés du même La question se pose dans la mesure où cette catégorie n'englobe pas l'ensemble des acteurs du sauvetage de Juifs durant la Shoah.

D’une part, des Juifs ont été sauvés par des Juifs eux-mêmes. Ainsi, alors que plusieurs citoyens suisses (et aussi français) ont été honorés du titre de Juste pour avoir fait passer ou aider à faire passer des Juifs de France en Suisse, des organisations juives de résistance ont également organisé de tels passages. À Budapest, de jeunes sionistes, les Chalutzim, ont joué un rôle très important dans les opérations de protection et de sauvetage des Juifs aux côtés de Carl Lutz et de son équipe, en particulier par la distribution de documents de protection. Cette dimension juive ressort bien de l'exposition mise sur pied par la Fondation Lutz de Budapest, présidée par un survivant. Elle est en revanche largement absente du récent film suisse consacré à $\mathrm{Lut}^{10}$. Se . Llle est en revanche largement absente du récent film suisse consacre a Lutz. Selon qưon presente à des elèves

D’autre part, des non-Juifs ayant porté secours à des Juifs n'ont pas été honorés du titre de Juste pour une raison ou pour une autre. Leurs actes peuvent paraître tout autant méritoires. Un cas parmi d'autres est celui du Suisse engagé dans une œuvre d'entraide dans le Sud de la France, honoré du titre de Juste pour avoir remis son passeport à un enfant juif qui allait tenter de passer la frontière suisse - même si cet enfant n'a finalement pas utiliséle document d'identité et qu'il a ensuite toujours refusé de témoigner en sa faveur auprès de Yad Vashem ; en revanche, un couple suisse qui avait hébergé ce même enfant dans sa ferme voisine et avait voulu lui remettre une pièce d'identité - ce que l'enfant a refusé -, n’a quant à lui, pas été honorélll (Wisard, 2007, 29).

À travers cet exemple - il y en aurait bien d'autres - on voit que l'élève peut passer à côté d’éléments décisifs si l'attention porte sur les seuls Justes parmi les
Nations. Ce risque concerne aussi bien l'interprétation des actes de sauvetage que l'évaluation des mérites de chacun, évaluation que l'élève effectue très certainement.

Élargir l'enseignement à d'autres actes de sauvetage que ceux des seuls Justes offre un intérêt incontestable. Cette démarche ne saurait toutefois conduire à mettre tous les actes de sauvetage sur un pied d'égalité. En effet, la figure du Juste comporte trois éléments tout à fait prometteurs dans un contexte pédagogique les Justes ont pris des risques, ils ont agi de manière désintéressée, ils ont porté secours à l'Autre, une personne dont ils ne partagent pas la religion.

En simplifiant, on dira qu'il s'agit d'actes de courage altruistes qui n'ont rien d'évident ni de spontané. Ils diffèrent ainsi, par exemple, des actes de certains passeurs qui ont certes sauvé des vies, mais ont dépouillé leurs victimes, ou encore du père ou de la mère qui a risqué sa vie pour sauver son enfant.

Deux cas de figure se présentent: le (futur) sauveteur connaît la personne, d'une autre religion, à qui il décide de porter secours, ou alors il ne la connaît pas. Ces deux cas de figure se retrouvent parmi les Justes de nationalité suisse qui ont hébergè des Juifs en France : la servante qui a pris en charge les enfants de la famille juive sauver le jeune Juif inconnu venu frapper à sa porte pendant la nuit.

L'acte de courage altruiste dont le Juste est le symbole invite les élèves à réfléchir sur les choix possibles, sur une marge de manœuvre - probablement plus étendue qu'on ne pourrait l'imaginer - dans des situations tragiques.

\section{LA NOTION DE " JUSTE SUISSE " OU CELLE DE " JUSTE FRANÇAIS "} EST-ELLE ÉVIDENTE ET PERTINENTE ?

La première question semblait naturelle, tant sont évidentes les limites d'une approchequi ne prendraiten compte que les Justes La deuxième question apparât incontestablement plus surprenante, voire même incongrue.

Yad Vashem, on le sait, publie des statistiques par pays des Justes qu'il a honorés. Or comment traiter les double-nationaux, les pays dont les frontières ont été modifiées, ou encore les personnes dont la nationalité a changé entre le moment de l'acte de sauvetage et celui de la décision de Yad Vashem?

S’agissant de la Suisse, un autre problème se pose. La majorité des Justes de nationalité suisse ont été honorés pour des actions réalisées en France. La plupart d'entre eux figurent dans la liste « Suisse » de Yad Vashem. Mais 20 autres apparaissent sur la liste « France » ${ }^{12}$. Pour l'anecdote, mentionnons que le premier nom qui figure sur la liste « Suisse » est celui d'une personne qui a eu plusieurs nationalités, mais jamais a nationalité suisse, pays ou il est certes né, mais qu'li a quitté définitivement à l’ấge de deux ans.

Dans ce contexte, établir une liste complète et cohérente de « Justes suisses » a très peu de sens. Il est préférable et scientifiquement plus pertinent de dresser

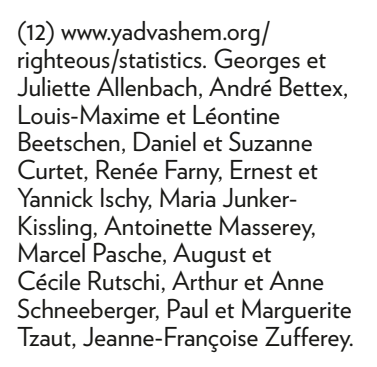


plusieurs listes : les Justes possédant la nationalité suisse au moment du sauvetage, ceux qui la possédaient lors de la décision de Yad Vashem, les double-nationaux, les étrangères devenues suisses par mariage et les Suissesses ayant perdu leur nationalité par mariage, enfin des Justes ayant un lien très indirect avec la Suisse, comme le premier nom figurant sur la liste « Suisse » de Yad Vashem. Bien entendu, additionner simplement les noms se trouvant sur les listes n’aurait aucune crédibilité.

Cette réflexion montre l'importance d'une approche transnationale et la nécessité de bien reconstituer les contextes historiques dans lesquels les actes sont intervenus. Les risques encourus pour le sauvetage étaient considérablement plus élevés en Pologne (peine de mort) qu'en Suisse par exemple, ou même en France (sur la question des différences entre les risques encourus, voir notamment Cabanel, 2012 p. 97-102)

Faire réfléchir des élèves sur ces actes de courage altruiste que constituent les actions de tous les Justes - mais également d'autres personnes - demande aussi de mettre en évidence les différences entre chacun de ces actes. Cela conduit à la dernière question, celle de l'exemplarité et de la représentativité des Justes.

\section{EXISTE-T-IL DES FIGURES EXEMPLAIRES DE JUSTES PARMI LES NATIONS ?}

La réponse est bien évidemment affirmative. Il existe des figures « exemplaires». Qưon songe simplement à Raoul Wallenberg, Oskar Schindler, ou encore Aristides de Sousa Mendes, ou, s’agissant de la Suisse, à Carl Lutz et à Paul Grüninger ${ }^{13}$. Quelles ont été les actions des deux Suisses ? ? $^{4}$

Carl Lutz a travailléà la légation ${ }^{15}$ de Suisseà Budapest de 1942 à la fin de laguerre. Il y dirigeait la division des intérêts étrangers et gérait à ce titre la représentation des intérêts britanniques, américains et ceux d'une dizaine d'autres Etats, auprès du gouvernement hongrois, puis, après mars 1944, également auprès de l'occupant allemand Le mandatbritannicur allemand Le ine. Surlabase de ce mandat et à partir d'une émigration légale jusqu’à mars 1944, Carl Lutz, avec l'aide d'autres personnes, a développé un système de protection diplomatique en faveur de milliers de Juifs. Ce système comprenait des lettres et passeports de protection, ainsi que des immeubles. En 1948, l'ancien président de l'organisation sioniste hongroise a adressé à Lutz une lettre de remerciements dans laquelle il écrit que ce dispositif a permis de sauver 62000 Juifs de Budapest ${ }^{16}$.

Paul Grüninger, quant à lui, a dirigé les forces de police du principal canton frontalier de l'Autriche avant et après l'Anschluss. Il est crédité d'avoir sauvé des

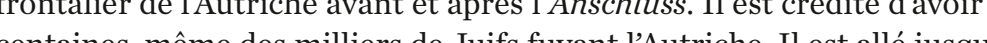
作 des documents, ce qui lui a valu d'être non seulement démis de ses fonctions, mais encore condamné pénalement.

\section{(1) 1950.} à Lutt, 24.12.1948, Archiv für
Zeitgeschichte, Zürich, Nachlass Zutz.
Figures exemplaires, oui sans aucun doute. Figures représentatives, assurément non. Lutz et Grüninger étaient des fonctionnaires, actifs en Suisse et en Hongrie. Or, sur la septantaine de Justes possédant la nationalité suisse durant la Shoah, la grande majorité ont été actifs en France et la moitié d'entre eux étaient des femmes. De plus, la proportion d'hommes et de femmes d'Église était notablement plus élevée que celle de fonctionnaires.

Se focaliser sur des grandes figures déforme donc le tableau d'ensemble. Un cond problèmestliéàl'utilisation quasisystématique du termede hóros ain second proble surestiè par exemple dans le titre du dernier film sur Lutz. Incontestablement, les Justes ont fait preuve d'un très grand courage. Il n'en demeure pas moins que certains d'entre eux ne se considèrent nullement comme des héros et qu'ils ne tiennent pas à lêtre. Comment ne pas être ému par ce témoignage de la fille d'un pasteur suisse honoré par Yad Vashem: « J'ai oublié une partie des noms et des faits que mon père citait, et cétait très rare qu'il le fasse. Ce qu'il a noté après la guerre, quelques heures avant sa mort, il nous a demandé à mon mari et à moi de tout brûler. » En bref, bon nombre de Justes considéraient leur action comme "normale », allant de soi.

Dès lors, se focaliser sur les grandes figures, sur les «héros » aux actions « extraordinaires» entraînele risque quelesélèves pensent que cetidéal restera de toute manière inatteignable, voire que les actions similaires qu'ils pourraient entreprendrè̀' inter

Se focaliser sur les « figures» de Justes comporte un autre danger. Celui de faire l'impasse sur le contexte historique, voire sur la dimension collective de certaines actions. Carl Lutz, par exemple, a agi avec le soutien de jeunes sionistes - on l'a vu -, mais aussi de collaborateurs de la légation de Suisse. Trois d'entre eux, dont l'un a été comme Raoul Wallenberg capturé par les Soviétiques, ont été honorés du titre de Juste. Ils n’apparaissent toutefois pas dans le film Carl Lutz, le héros oublié.

L’organisation qui m’a demandé d'écrire un livre sur les Justes suisses envisageait a l'origine uniquement une série de notices biographiques, à savoir une golorie de portraits. Je n'ai cceptéle mandat qu’à condition d'adopter un approche thé de 作 tique, autrement dit de replacer les actions des Justes dans leur contexte historique,
détablir un bilan historiographique et d'indiquer les principales sources.

Aborder le thème des Justes ne saurait faire l’impasse d'une réflexion sur les sources. Les traces écrites contemporaines sont rares, voire souvent inexistantes, en raison de la nature même de ces activités, illégales et risquées. Les dossiers de Yad Vashem contiennent généralement les témoignages, ultérieurs, de la ou des personnes sauvées et documentent avant tout le moment du sauvetage, laissant dans l'ombre le contexte historique ou encore la vie du Juste ou celle de la personne sauvée. Dans certains cas, nous disposons de davantage d'informations écrites. In n'en reste pas moins indispen 作 reste exposée au risque d'hagiographie.

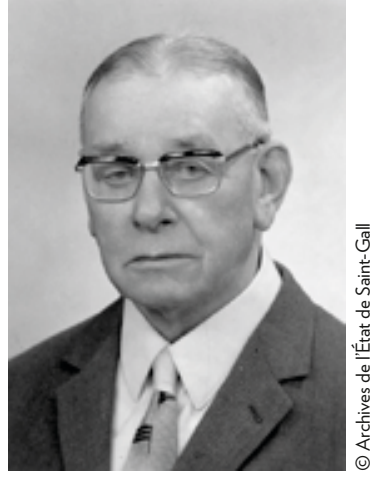

- Paul Grüninger, saint-galloise au momen de l'Anschluss de l'Autriche, 
Les Justes parmi les Nations dans un context éflexions à partir du cas suisse (suite)

Examinons un dernier point, toujours en lien avec la question de l'exemplarité Se limiter à des grandes figures n'est pas satisfaisant, on l'a vu. Du reste, Yad Vashem n'opère aucune distinction en décernant les titres. Qu’une seule personne ou que plusieurs milliers de personnes aient été sauvées par un Juste n’entraîne aucune différence. Le titre est le même, la médaille également. Celle-ci contient le message suivant : « Quiconque sauve une vie, sauve l'univers entier. » C'est donc l'acte de sauvetage qui est honoré, et non pas le nombre de personnes sauvées.

Une telle approche est indéniablement salutaire, car il est indispensable de set le sauvetage d'un seul peut se révéler bien plus risqué que celui de plusieurs. Mettre l'ensemble des Justes sur le même pied comporte toutefois deux dangers. Le premier, on l'a vu, est liéà la frontière, parfois pour le moins discutable, entre Justes et personnes qui n’ont pas été honorées malgré des actions aussi méritoires. Le second danger serait de perdre de vue les proportions, voire d'evacuer complètement les chiffres. Avoir sauvé des centaines de Juifs dans un pays occupé d'Europe de l'Est n'est quand même pas la même chose quavoir laissé entrer en Suisse une famille juive.

Lecriture de l'histoire requiert toujours des mises en contexte et des proportions. . à s'en souvenir. I

-Juifs se pressant devant
la Maison de verre, "Département d'émigration

Budapest., 1944

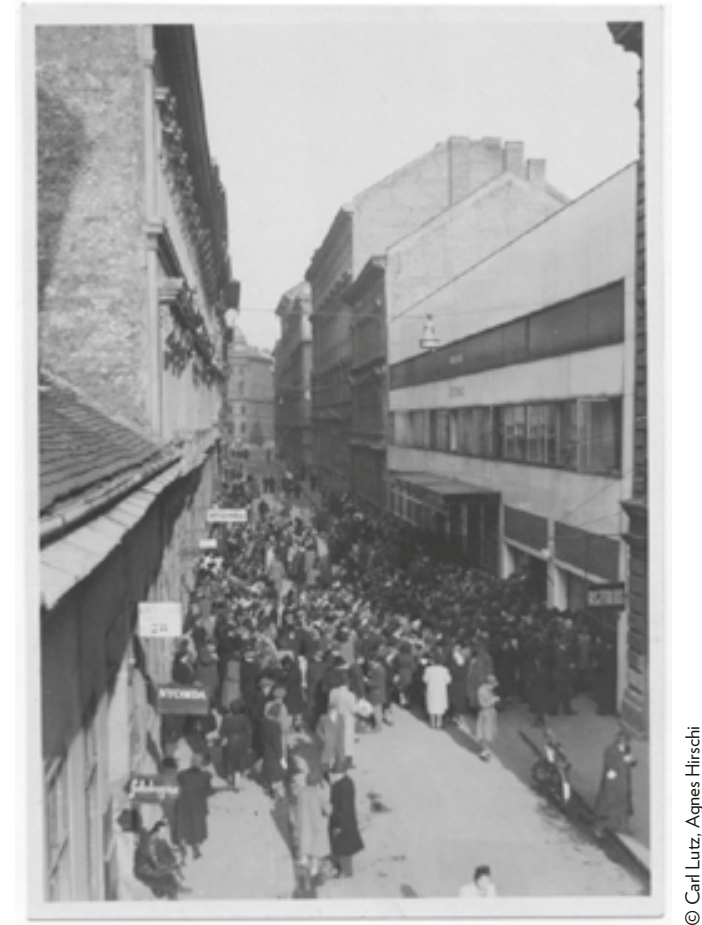

BIBLIOGRAPHIE

- Alejandro Baer et Pedro Correa Martin-Arroyo, Pedro, « The Politics of Holocaust Rescue Myths in Spain. From Francoist Humanitarianis
to the Righteous Diplomats , in Corry Guttstadt, e. a. (dir.), Bystanders, Rescuers or Perpetrators? The Neutral Countries and the

Arieh Ben-Tov, Facing the Holocaust in Budapest: The International Committee of the Red Cross and the Jews in Hungarl/
Dordrecht/Boston, Martinus Nijhoff/Norwell, 1988 .

- Arieh Ben-Tov, Das Rote Kreuz kam zu spät: Die Auseinandersetzung zwischen dem, judischen Volk und dem Intereratitionalen Komitee vom
Roten Kreuz im Zweiten Weltkrieg: Die Ereignisse in Ungarn, Zürich.

- Arieh Ben-Tov, Face au génocide. La Croix-Rouge et les Juifs de Hongrie,
1941-1945, Lausanne Payot, 1997.

- Friedel Bohny-Reiter, Journal de Rivesaltes 1941-1942, Genève, Zo

- Friedel Bohny-Reiter, Vorhof der Vernichtung: Tagebuch einer Schweizer Schwester im franzosischen Intern.
Konstanz, Hartung-Gorre, 1995.

- Patrick Cabanel, Histoire des Justes de France, Paris, Armand Colin,

- Sarah Gensburger « La diffusion internationale de la catégorie de
"Juste parmi les Nations" (R) Penser larticulatetion entre diffusion des droits de l'homme et globalisation de la mémoire », Revue - Alexander Grossman, Nur das Gewissen. Carl Lutz und seine Budapester
Aktion. Geschichte und Portrat, Waldgut, Wald, 1988. - Anne-Marie Im Hof-Piguet, La Filìre en France occupée 1942-1944.
Yverdon-les-Bains, Ia Thièle, 1985.

- Anne-Marie Im Hof- Piguet, Fluchtweg durch die Hinterturi: Eine
Rotkreuz-Helferin im besestzen Frankreich $1942-1944$, Frauenfeld, Im Rotkreuz-Helferii

- Marco Jorio, , Judenretter Carl Lutz „, NZZ Geschichte, (7),

- Stefan Keller, Grüningers Fall: Geschichten von Flucht und Hilfe, Zurich,

- Stefan Keller, Délit d'humanité. L'affaire Grüninger, Lausanne, Ed. d'en

- Beate Kosmala et Revital Ludewig-Kedmi, Verbotene Hilfe. Deutsche 2003

- Mediendossier, Carl Lutz, der vergessene Held. Ein Kino-
Dokumentarfilm von Daniel von Aarburg, 2014, p. 10.

- Marcel Pasche, Années de guerre et de fraternité, Le Mont-sur-

Lausanne, Ouverture, 1995

- Erika Rosenberg, Carl Lutz und d do

Sebastian Steiger, Die Kinder von Schloss La Hille, Basel, Brunnen-

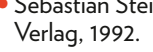

Steiger Les enfants duchâteau de La Hille, Bâle, Brunnen,

- Theo Tschuy, Carl Lutz und die Juden von Budapest, Zürich, Verlag
Neue Zürcher Zeitung, 1995.

- Theo Tschuy, Diplomatie dangereuse. Carl Lutz, l'homme qui a sauvé les

- Theo Tschuy, La casa di vetro. Storia di Carl Lutz, lo svizzero che salvò

- György Vámos, Carl Lutz (1895-1975). Diplomate suisse à Budapest en
1944. Un Juste parmi les Nations, Gollion, Infolio, 2012.

- François Wisard, Les Justes suisses. Des actes de courage méconnus au 